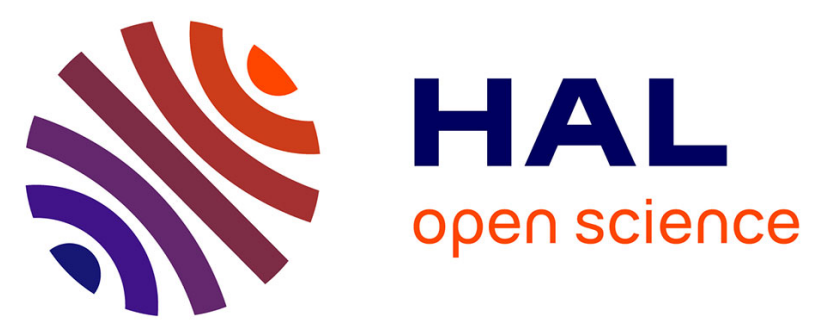

\title{
Comparaison de pondeuses mi-lourdes naines ( $d w)$ ou normales avec deux aliments de taux protéique et énergétique différents
}

G. Coquerelle, P. Mérat, M. Boitard

\section{- To cite this version:}

G. Coquerelle, P. Mérat, M. Boitard. Comparaison de pondeuses mi-lourdes naines (dw) ou normales avec deux aliments de taux protéique et énergétique différents. Productions Animales, 1990, 3 (1), pp.17-19. hal-00895885

\section{HAL Id: hal-00895885 \\ https://hal.science/hal-00895885}

Submitted on 1 Jan 1990

HAL is a multi-disciplinary open access archive for the deposit and dissemination of scientific research documents, whether they are published or not. The documents may come from teaching and research institutions in France or abroad, or from public or private research centers.
L'archive ouverte pluridisciplinaire HAL, est destinée au dépôt et à la diffusion de documents scientifiques de niveau recherche, publiés ou non, émanant des établissements d'enseignement et de recherche français ou étrangers, des laboratoires publics ou privés. 


\section{G. COQUERELLE, P. MERAT, M. BOITARD}

Avec la collaboration technique de J.-P. NOE

INRA Laboratoire de Génétique factorielle 78350 Jouy-en-Josas

\section{Comparaison de pondeuses mi-lourdes naines $(d w)$ ou normales} avec deux aliments de taux protéique et énergétique différents

Chez la poule, l'intérêt du gène de nanisme lié au sexe découle de la réduction de l'ordre de $30 \%$ du poids corporel qu'il entraîne chez la pondeuse reproductrice adulte, dont la consommation alimentaire est ainsi diminuée de 20 à $25 \%$, alors que le poids moyen des oeufs n'est réduit que de 5 à $10 \%$. L'effet dépressif sur le nombre d'oeufs, absent chez les souches « chair », est relativement modéré pour des pondeuses mi-lourdes.

Chez les reproductrices de type " chair », les mêmes aliments peuvent être distribués aux poules naines et aux poules normales (Larbier 1985). En revanche, chez les poules légères de type Leghorn, le taux protéique de l'aliment doit être plus élevé pour assurer une ponte optimale (Guillaume 1976). Nous avons voulu examiner la réponse à cet égard de poules naines de taille intermédiaire (type " oeuf brun ») pour lesquelles, à notre connaissance, on a peu d'informations.

\section{Résumé}

Cent vingt huit poules provenant d'une population de type oeuf brun mi-lourde, les unes de taille normale $\left(D w^{+}\right)$, les autres naines $(d w)$ ont été réparties à 16 semaines en cages individuelles. Chaque génotype a été réparti en deux groupes, alimentés l'un avec un régime à $15,7 \%$ de protéines totales et $2650 \mathrm{kcal} \mathrm{EM} / \mathrm{kg}$, l'autre à $17,6 \%$ de protéines totales et $2750 \mathrm{kcal} \mathrm{EM} / \mathrm{kg}$. Les performances de ponte jusqu'à l'âge de 43 semaines ont été légèrement plus faibles pour le génotype $d w$ : de l'ordre de $5 \%$ pour le nombre d'oeufs, 6-7\% pour le poids moyen des oeufs. L'indice de consommation a été plus faible pour le génotype $d w$, de respectivement $20 \%$ et $14 \%$ aux âges de 27 et 41 semaines. Le poids corporel a augmenté davantage (avec le régime 1) et le poids des oeufs relativement moins avec l'âge, pour les poules $D w^{+}$que pour les poules $d w$. En fin de contrôle, les poules à plumage détérioré ont été moins fréquentes parmi les naines que parmi les normales. L'aliment le moins riche a eu tendance à augmenter la consommation, le poids corporel et le nombre d'oeufs de façon plus marquée pour les poules normales que pour les naines. Les jours de " pauses " ont été plus fréquents pour les poules normales que pour les naines avec le régime plus riche. L'effet du gène $d w$ sur l'indice de consommation a été sensiblement le même pour les deux régimes, malgré un moindre avantage des poules $d w$ avec l'aliment moins riche à l'âge de 41 semaines.

\section{1 / Conditions expérimentales}

En avril 1987, un croisement de type pondeuse mi-lourde à oeufs bruns, issu de 9 pères hétérozygotes $D w^{\prime} d w$ pour le gène de nanisme lié au sexe et de 44 mères, a donné en une éclosion unique des descendantes normales $\left(D w^{+}\right)$ et naines $(d w)$, qui ont été élevées au sol. Elles ont reçu, à volonté jusqu'à 10 semaines, un aliment contenant $19,8 \%$ de protéines totales et $2800 \mathrm{kcal} / \mathrm{kg}$ d'énergie métabolisable (EM) calculée, et, de 10 à 16 semaines, un aliment à $15,3 \%$ de protéines totales et $2750 \mathrm{kcal} \mathrm{EM} /$ $\mathrm{kg}$. A l'âge de 16 semaines, elles ont été mises en cages individuelles. A partir de cet âge, chaque génotype dans chaque famille de père a été réparti en deux groupes recevant chacun un aliment différent, l'un à $15,5 \%$ de protéines totales, $2650 \mathrm{kcal} \mathrm{EM} \mathrm{/} \mathrm{kg} \mathrm{(aliment} \mathrm{1),} \mathrm{l'autre} \mathrm{à}$ $17,6 \%$ de protéines totales et $2750 \mathrm{kcal} / \mathrm{kg}$ (aliment 2). Les principales caractéristiques nutritionnelles des deux aliments sont données au tableau 1. Les poulettes étaient éclairées 10 heures par 24 heures en poussinière et 14 heures par 24 heures à partir de 16 semaines.

Les contrôles de ponte ont été effectués jusqu'à l'âge de 43 semaines. Les variables suivantes étaient enregistrées individuellement: âge au $1^{1 r}$ oeuf; nombre d'oeufs pondus pendant la période de contrôle ; intensité de ponte correspondante (rapport du nombre d'oeufs au nombre de jours de contrôle, "pauses" de plus de 2 jours déduites) ; jours de " pause » en pourcentage du nombre total des jours de contrôle; pourcentages d'oeufs cassés et d'oeufs « mous» (sans coquille). 
Tableau 1. Composition des régimes.

\begin{tabular}{|l|c|c|}
\hline \multicolumn{1}{|c|}{ Caractéristique calculée } & Régime 1 & Régime 2 \\
\hline Protéines totales (\%) & 15,70 & 17,60 \\
Matières grasses (\%) & 3,50 & 3,50 \\
Calcium (\%) & 3,35 & 3,10 \\
Phosphore disponible (\%) & 0,35 & 0,40 \\
Méthionine (\%) & 0,31 & 0,29 \\
Lysine (\%) & 0,67 & 0,80 \\
Energie métabolisable kcal/kg & 2650 & 2750 \\
\hline
\end{tabular}

Au cours de deux périodes de 28 jours lla première entre les âges de 25 et 29 semaines, la seconde entre 39 et 43 semaines), le poids corporel moyen et la masse d'oeufs pondus ont été enregistrés individuellement et la consommation alimentaire relevée par groupes de 18 poules du même génotype recevant le même type d'aliment. L'indice de consommation moyen par génotype en était déduit à chaque âge. Le poids moyen des oeufs est également donné par groupe pour chaque période.

\section{Analyse statistique}

Les nombres d'animaux contrôlés par groupe figurent au tableau 2. Pour les variables mesurées individuellement, les effets du génotype, du régime et l'interaction génotype $x$ régime ont été testés par analyse de variance tenant compte des effectifs légèrement inégaux dans les sous-groupes. Par contre, disposant seulement de 2 répétitions de chaque combinaison génotype-régime pour la consommation alimentaire, l'indice de consommation et le poids moyen des oeufs, nous n'avons pas fait d'analyse statistique pour ces variables.

\section{2 / Résultats et discussion}

A partir de l'âge de 16 semaines, les nombres de mortes parmi les poules $D w^{\prime}$ et $d w$ avec les régimes 1 et 2 ont été respectivement $0,4,3$ et 5. Aucun effet du génotype ni du régime sur la mortalité n'est significatif. Le tableau 2 montre les valeurs moyennes par génotype et régime pour les variables mentionnées ci-dessus, et les analyses de variance correspondantes.

Tableau 2. Paramètres de ponte et d'efficacité jusqu'à l'âge de 43 semaines pour les poules normales et naines selon le régime.

\begin{tabular}{|c|c|c|c|c|c|c|c|c|}
\hline \multirow{2}{*}{ Variable } & \multicolumn{4}{|c|}{$\begin{array}{c}\text { Moyenne par régime et génotype } \\
\text { Régime } 1 \quad \text { Régime } 2\end{array}$} & \multicolumn{3}{|c|}{$\begin{array}{l}\text { Analyse de variance } \\
\text { Valeurs de } F \text { et signification }\end{array}$} & \multirow{2}{*}{$\begin{array}{l}\text { Ecarl } \\
\text { type } \\
\text { résiduel }\end{array}$} \\
\hline & $\begin{array}{c}D \boldsymbol{W}^{+} \\
(\mathrm{n}=\mathbf{3 4})\end{array}$ & $\begin{array}{c}d w \\
(n=32)\end{array}$ & $\begin{array}{c}D \boldsymbol{w}^{+} \\
(\mathrm{n}=\mathbf{3} 1)\end{array}$ & $\begin{array}{c}d w \\
(\mathrm{n}=31)\end{array}$ & $\begin{array}{l}\text { Génotype } \\
\text { (G) }\end{array}$ & $\underset{\text { (R) }}{\text { Régime }}$ & G X R & \\
\hline Age au 1"r ouf (i) & 137.8 & 142,9 & 140,8 & 142,1 & $2,96^{\circ}$ & 0.35 & 1,12 & 10,3 \\
\hline Nombre d'œufs & 138,0 & 126,3 & 126,1 & 125,6 & 3,44 & 3,62 & 2,92 & 18,5 \\
\hline Intensité de ponte $(\%)$ & 82,1 & 78,8 & 80,6 & 76,1 & $13,02^{\star * *}$ & $3,66^{\prime}$ & 0,25 & 6,2 \\
\hline Jours de pauses $[\%]$ & 7,8 & 9,4 & 13,0 & 7,1 & 1,48 & 0,71 & $4,72^{\star}$ & 9,8 \\
\hline CEufs cassés [\%] & 4,5 & 1,5 & 6,5 & 1,0 & $8,99^{\star *}$ & 0,27 & 0,77 & 8,1 \\
\hline CEuis mous $(\%)$ & 5,3 & 1,1 & 5,7 & 0,6 & $48,09^{* * *}$ & 0,0 & 0,40 & 3,8 \\
\hline $\begin{array}{l}\text { (à } 27 \text { semaines) } \\
\text { Poids corporel (g) }\end{array}$ & 2476 & 1725 & 2363 & 1661 & $229,30^{\star * *}$ & $5,57^{*}$ & 1,10 & 265 \\
\hline (à 41 semaines) & 2927 & 1952 & 2651 & 1952 & $191,51^{\star * *}$ & $3,12^{+}$ & 2,69 & 354 \\
\hline De 25 à 29 semaines : & & & & & & & & \\
\hline $\begin{array}{l}\text { Masse d'œufs (g/28 j.) } \\
\text { Poids moyen }\end{array}$ & 1147 & 1051 & 1026 & 980 & 3,08 & $6,67^{* *}$ & 0,01 & 190 \\
\hline $\begin{array}{l}\text { des oufs (g) } \\
\text { Aliment consommé }\end{array}$ & 50,9 & 47,2 & 50,3 & 46,5 & & & & \\
\hline$(\mathrm{g} / 28 \mathrm{j})$. & 4072 & 2929 & 3637 & 2798 & & & & \\
\hline Indice de consommation & 3,55 & 2,79 & 3,54 & 2,85 & & & & \\
\hline De 39 à 43 semaines : & & & & & & & & \\
\hline $\begin{array}{l}\text { Masse d'œufs }(\mathrm{g} / 28 \mathrm{j} .] \\
\text { Poids moyen }\end{array}$ & 1146 & 912 & 1004 & 998 & $8,14^{\star *}$ & 0,44 & 3,00 & 238 \\
\hline $\begin{array}{l}\text { des azufs (g) } \\
\text { Aliment consommé }\end{array}$ & 58,9 & 55,5 & 60,1 & 57,0 & & & & \\
\hline$(\mathrm{g} / 28 \mathrm{j})$. & 4122 & 3026 & 3529 & 2891 & & & & \\
\hline Indice de consommation & 3,60 & 3,20 & 3,51 & 2,90 & & & & \\
\hline
\end{tabular}

Nombre de degrés de liberté : 1 pour les effets du génotype $(\mathrm{G})$. du régimes (R) et de l'interaction génotype $\times$ régime $(\mathrm{G} \times \mathrm{R}) ; 124$ pour la variance résiduelle ${ }^{+}{ }^{*},{ }^{* *} .{ }^{* \star *}$, respectivement $\mathrm{P}<0,10 ; \mathrm{P}<0,05 ; \mathrm{P}<0,01 ; \mathrm{P}<0,001$ 
Nous retrouvons des effets associés au gène $d w$ maintes fois décrits : réduction du poids corporel de l'ordre de $30 \%$, abaissement du pourcentage d'oeufs cassés et d'oeufs "mous » sans coquille. Dans cette population de type " oeuf brun" relativement lourde, la diminution des performances de ponte due au gène $d w$ est limitée : de l'ordre de $5 \%$ pour le nombre d'oeufs jusqu'à 43 semaines, 6-7\% pour le poids moyen des oeufs, entre 5 et $10 \%$ pour la masse d'oeufs. Du fait de la réduction de la consommation d'aliment, il en résulte une amélioration de l'indice de consommation par comparaison avec les poules $D w^{+}$de même origine, voisine de $20 \%$ dans la première période de contrôle et de $14 \%$ dans la seconde. Ces observations s'inscrivent dans la ligne d'un effet défavorable sur la production d'oeufs d'autant plus réduit et d'un effet favorable sur l'efficacité alimentaire d'autant plus accentué que le gène $d w$ est introduit dans une population de plus grande taille (Mérat 1990). Au cours du temps, le poids corporel des poules $d w$ augmente moins que celui des poules normales avec le régime 1 , leur poids moyen d'oeufs croît davantage (respectivement de 15,7 et $17,6 \%$ de la période 1 à la période 2 pour les poules $D w^{*}$ et $d w$ recevant le régime 1 ; de 19,5 et $22,6 \%$ avec le régime 2). Par contre, il n'y a pas d'effet différentiel évident de l'âge sur les deux génotypes pour la masse d'oeufs et l'indice de consommation. On peut signaler qu'en fin de contrôle il y a eu moins de poules ayant des zones déplumées plus ou moins étendues parmi les naines quel que soit le régime (au total 24 sur 66 survivantes pour le génotype $D w^{+}$et 8 sur 63 pour le génotype $d w, \mathrm{P}<0,01$ ), ce qui peut être un élément favorable à l'efficacité alimentaire.

La consommation du second régime, plus riche en protéines et en énergie, est globalement inférieure à celle du premier. Ceci s'accompagne d'un poids corporel un peu plus faible, sans effet décelable sur le poids moyen des oeufs. Par contre, quoique moins riche, le premier régime est associé à un nombre d'oeufs et à une intensité de ponte un peu plus élevés, de même qu'à une masse d'oeufs plus importante en première période.

La seule interaction significative entre génotype et régime concerne le pourcentage de jours de "pauses", peu différent entre génotypes en présence du premier régime, mais plus élevé pour les poules $D w^{\prime}$ en régime 2 . Nous n'avons pas d'explication de cet effet. Sans que l'interaction génotype x régime sur le nombre d'oeufs jusqu'à 43 semaines soit significative, ce nombre apparaît supérieur chez les poules $D w^{+}$en régime 1, alors qu'il est presque le même pour les deux génotypes en régime 2 . Le surcroît de consommation alimentaire en présence du régime 1 est plus marqué pour le génotype $D w^{+}$et ceci semble s'accentuer en période 2. En fin de compte, l'effet du génotype sur l'indice de consommation est pratiquement identique pour les deux régimes en première période, un avantage de ponte plus marqué du génotype $D w^{+}$en régime 1 étant compensé par un excès d'ingestion alimentaire. L'avantage des poules naines pour l'efficacité alimentaire paraît réduit en présence du premier régime dans la seconde période, mais la signification de cet effet resterait à prouver. Au total, dans le contexte d'une souche de format mi-lourd, les poules nanifiées n'apparaissent pas sensiblement désavantagées en présence d'un aliment moins riche.

\section{Remerciements}

Nous sommes redevables à la Coopérative Agricole du Hurepoix ( 91470 Limours) de la réalisation des régimes expérimentaux qui a promis re tramil.

\section{Références bibliographiques}

GUILLAUME J., 1976. The dwarfing gene dw; its effects on anatomy, physiology, nutrition, management. Its application in poultry industry. Worlds's Poult. Sci. J., 32, 285-303.

LARBIER M.. 1985. Field restriction in dwarf and normal broiler breeders. 5th European Symposium on Poultry Nutrition, Maale Hashamisha, Israel, S. Bornstein ed. 56-64.

MERAT P., 1990. Pleiotropic and associated effects of major genes. In R.D. Crawford ed : Poultry Breeding and Genetics. Elsevier, Amsterdam. (sous presse).

\section{Summary}

Comparison of medium-heavy dwarf (dw) and normal layers using two feeds of different protein and energy levels.

One hundred and twenty-eight pullets issued from a medium heavy brown egg type population, either normal sized $\left(D w^{+}\right)$or $d w a r f(d w)$, were housed in individual cages at the age 16 weeks. Each genotype was divided into 2 groups, each receiving one of two commercialtype feeds, either having $15.7 \%$ crude protein and $2650 \mathrm{kcal} \mathrm{ME} / \mathrm{kg}$, or $17.6 \%$ crude protein and $2750 \mathrm{kcal} \mathrm{ME} / \mathrm{kg}$. Laying performance till the age 43 weeks was slightly decreased by the $d w$ gene compared to $D w^{+}$(about $5 \%$ for egg number, 6-7 \% for mean egg weight). The dwarf gene gave a gain in feed conversion with respect to egg production (respectively $20 \%$ and $14 \%$ at the ages of 27 and 41 weeks). With advancing age, there was a greater increase in mean body weight and mean egg weight increased relatively less for $D w^{+}$than for $d w$ hens when fed with the higher protein and energy level feed. At the end of the control period, there were less hens with deteriorated plumage among dwarf than among normal females.

The poorer diet increased feed consumption, body weight and egg number, this effect tended to be more marked for normal than for dwarf females. "Pause" days were more frequent for $D w^{+}$than for $d w$ females on the richer diet. The effect of the $d w$ gene on feed conversion showed little difference between the two diets, although decreased conversion by $d w$ hens on the poorer diet was suggested in the second period of control.

COQUERELLE G, MERAT P, BOITARD M., 1990. Comparaison de pondeuses mi-lourdes naines $(\mathrm{dw})$ ou normales avec deux aliments de taux protéique et énergétique différents. INRA Prod. Anim., 3(1), 17-19. 